\title{
SOHO/SUMER observations of prominence oscillation before eruption
}

\author{
P. F. Chen ${ }^{1}$, D. E. Innes ${ }^{2}$, and S. K. Solanki ${ }^{2}$ \\ 1 Department of Astronomy, Nanjing University, Nanjing 210093, PR China \\ e-mail: chenpf@nju.edu.cn \\ 2 Max-Planck-Institut für Sonnensystemforschung, 37191 Katlenburg-Lindau, Germany \\ e-mail: [innes; solanki] @mps.mpg.de \\ Received 8 February 2008 / Accepted 21 February 2008

\section{ABSTRACT}

\begin{abstract}
Context. Coronal mass ejections (CMEs), as a large-scale eruptive phenomenon, often reveal some precursors in the initiation phase, e.g., X-ray brightening, filament darkening, etc., which are useful for CME modelling and space weather forecasting. Aims. With the Solar Ultraviolet Measurements of Emitted Radiation (SUMER) spectroscopic observations of the 2000 September $26 \mathrm{CME}$, we propose another precursor for CMEs, namely, long-time prominence oscillations.

Methods. We observed the prominence oscillation-and-eruption event by ground-based $\mathrm{H} \alpha$ telescopes and space-borne white-light, Results. The observations indicate that a siphon flow was moving from the proximity of the prominence to a site at a projected distance of $270^{\prime \prime}$, which was followed by repetitive $\mathrm{H} \alpha$ surges and continual prominence oscillations. The oscillation lasted 4 hours before the prominence erupted as a blob-like CME. The analysis of the multiwavelength data indicates that the whole series of processes fits well into the emerging flux trigger mechanism for CMEs. In this mechanism, emerging magnetic flux drives a siphon flow due to increased gas pressure where the background polarity emerges. It also drives $\mathrm{H} \alpha$ surges through magnetic reconnection where the opposite polarity emerges. The magnetic reconnection triggers the prominence oscillations, as well as its loss of equilibrium, which finally leads to the eruption of the prominence. It is also found that the reconnection between the emerging flux and the pre-existing magnetic loop proceeds in an intermittent, probably quasi-periodic, way.
\end{abstract} \\ EUV imaging, and spectroscopic instruments. In particular, the SUMER slit was observing the prominence in a sit-and-stare mode.
}

Key words. Sun: coronal mass ejections (CMEs) - Sun: prominences - Sun: oscillations - Sun: magnetic fields

\section{Introduction}

Coronal mass ejections (CMEs) are large-scale phenomena on the Sun, which may drive major magnetic storms on Earth, and hence have been under extensive investigation in the context of space weather for decades. CMEs often present thermal or nonthermal signatures before their eruptions, which are called precursors. The precursors of a CME are very useful since they can be used to predict the occurrence of a CME, as well as to construct CME initiation models (see Gopalswamy et al. 2006, for a review).

The precursors found in the past decades can be summarised as follows: (1) Emerging flux: Feynman \& Martin (1995) found that two thirds of CMEs associated with quiescent filaments occurred after substantial amounts of new magnetic flux emerged in the vicinity of the filament. They also found that all filaments observed during a two-month period, in which the new flux was oriented favourably for reconnection, erupted. The merit of reconnection-favoured emerging flux as a precursor is that it appears a few days before the CME eruption, so that the eruption can be predicted a few days earlier. In order to interpret such a high correlation, Chen \& Shibata (2000) proposed an emerging flux trigger mechanism for CMEs where the reconnection between the coronal field and the emerging flux, either inside or outside the filament channel, restructures the coronal magnetic configuration, leading to the loss of equilibrium of the flux rope system. As a result, a current sheet forms below the flux rope. The ensuing reconnection of the current sheet results in the final eruption of the flux rope. Such a picture was confirmed by Sterling et al. (2005, 2007). (2) X-ray brightening: Harrison et al. (1985) studied $6 \mathrm{CME} /$ flare events and found that at a time coincident with the projected onset of the CMEs, there is a small, soft X-ray (SXR) enhancement $\sim 20$ min before the impulsive phase of the main flare. Such an SXR enhancement might correspond to the reconnection between the emerging flux and the preexisting magnetic field discussed above. (3) Radio noise storms: Lantos et al. (1981) found that intense radio noise storms appear before the eruption of a CME, which implies that the large-scale magnetic structure evolves before the final disruption. Ramesh \& Sundaram (2001), Chertok et al. (2001), and more recently, Wen et al. (2007) verified that CMEs are preceded by either the beginning or disappearance of noise storms. (4) Type-III radio burst groups: Jackson et al. (1978) studied 40 CMEs observed by the Skylab satellite and found that 5 to $10 \mathrm{~h}$ prior to the time when a CME is first visible, the occurrence rate of type-III radio bursts increases by at least 2.5 times the average. The result might also be understandable in the framework of the Chen \& Shibata (2000) initiation model, where localised reconnection in the trigger phase could accelerate electrons and produce type-III bursts well before the eruption of the CME. (5) Filament darkening and widening: Martin (1980) summarised preflare conditions and pointed out that filaments often become darker and wider about $1 \mathrm{hr}$ before the occurrence of a long duration flare, which is the type that is always accompanied by a CME.

In this paper, we present extreme ultraviolet (EUV) spectroscopic observations of oscillations of a prominence before its 
eruption, and propose that the long-time prominence oscillations are another precursor of CMEs. Note that a filament is called a prominence when it is located above the solar limb.

\section{Observations}

Two days after its first appearance, a prominence straddled over the east limb on 2000 September 25, with the leading part sitting on the solar disc as a filament. Starting from 20:50 UT on Sep. 25 and ending at 08:00 UT on Sep. 26, the slit of the Solar Ultraviolet Measurements of Emitted Radiation (SUMER) spectrometer on board the Solar and Heliospheric Observatory (SOHO) spacecraft was positioned across the prominence, and spectral data were recorded in the sit-and-stare mode. The $11 \mathrm{~h}$ spectroscopic observation covers the prominence eruption as well as its oscillation before the eruption. We also detected the dynamics of the prominence prior to the eruption by the $\mathrm{H} \alpha$ images from Big Bear Solar Observatory (BBSO) and Mauna Loa Solar Observatory (MLSO), while the activation and the ensuing eruption were observed by the EUV Imaging Telescope (EIT; Delaboudinière et al. 1995) with the Fe XII $195 \AA$ filter and the Large Angle and Spectrometric Coronagraph (LASCO; Brueckner et al. 1995) in white light, both being aboard the SOHO spacecraft.

The SUMER spectrometer (Wilhelm et al. 1995) is able to observe simultaneously any selected $40 \AA$ window within its 660-1600 A wavelength range. For the event analysed in this paper, emissions in the window 1098-1138 $\AA$ were obtained with a cadence of $169 \mathrm{~s}$ and a slit of $4^{\prime \prime} \times 300^{\prime \prime}$. It contained both cold and hot lines in the temperature range of $10^{4}-10^{6} \mathrm{~K}$, including the chromospheric line C I $1118.45 \AA(\sim 0.01 \mathrm{MK})$, and transition region line $\mathrm{S}$ III/Si III $1113 \AA(\sim 0.05 \mathrm{MK})$. The standard procedure, including calibrations and corrections, is applied in the data analysis.

BBSO recorded full-disc $\mathrm{H} \alpha$ images with a high cadence ( $1 \mathrm{~min}$ ) and a pixel size of $\sim 1^{\prime \prime}$. Full-disc $\mathrm{H} \alpha$ observations from MLSO were also examined for reference.

\section{Results}

The prominence, which was located above the solar east limb and $\sim 10^{\circ}$ to the south of the equator, extended to the northwest, where it became visible as a faint filament on the disc as illustrated by the $\mathrm{H} \alpha$ image in Fig. 1 (the filament is barely visible in the figure as the brightness was adjusted to best show the details of the prominence). The narrow rectangle in the figure indicates the position of the SUMER slit. At least from 15:18 UT on Sep. 25 when the first $\mathrm{H} \alpha$ image in BBSO is available, a flow of chromospheric material, presumably a siphon flow, was driven from the solar surface in the proximity of the prominence along an arc pointing to the south in the plane of the sky, probably guided by magnetic field lines. Passing through the prominence in the projected plane, the $\mathrm{H} \alpha$-emitting plasma drained down to the solar surface at a place about $270^{\prime \prime}$ to the south of the prominence, as shown in Fig. 2. The figure displays the time sequence of the $\mathrm{H} \alpha$ images, with a high cadence in the upper row to highlight the siphon flow and a low cadence in the lower row to show the longer-term dynamics. The pumping of the $\mathrm{H} \alpha$ siphon flow from its origin ended at $\sim 21: 32$ UT on Sep. 25. A time-slice analysis along the trajectory of the flow indicates that the plasma was moving with a speed of $\sim 60 \mathrm{~km} \mathrm{~s}^{-1}$, projected on the plane of the sky.

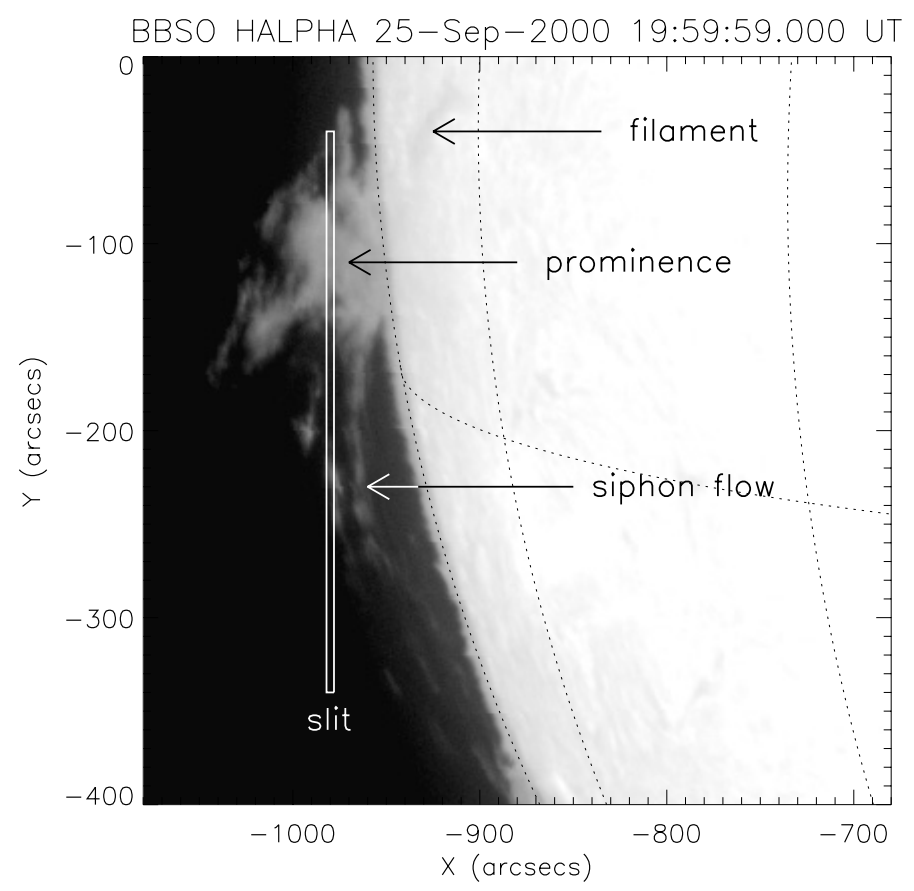

Fig. 1. H $\alpha$ image from BBSO showing the positions of the prominence and the SUMER slit. Note that a siphon flow is moving to the south of the prominence.

During the continual motion of the siphon flow, an $\mathrm{H} \alpha$ surge was seen to rise below the trajectory of the siphon flow at 20:27 UT, and reached its peak at 20:32 UT, as indicated by the ellipse in panel (d) of Fig. 2. We estimate the projected rise speed of the surge to be $\sim 30 \mathrm{~km} \mathrm{~s}^{-1}$. The H $\alpha$ surge then fell down to the chromosphere. About one hour later, i.e., at 21:26 UT, a second surge erupted at the same site, as indicated by the ellipse in panel (f). Another three surges erupted repetitively from the same site at $\sim 22: 26 \mathrm{UT}, \sim 22: 42 \mathrm{UT}$, and $\sim 23: 29 \mathrm{UT}$, respectively.

The spectroscopic observation of SUMER started at 20:50 UT on Sep. 25. The SUMER slit crossed the prominence, and coincidently, its lower part covered the trajectory of the $\mathrm{H} \alpha$ siphon flow, as seen in Fig. 1. In order to see the prominence with the SUMER data, the time evolution of the intensity of the cold line C I $1118.45 \AA$ is plotted in the left panel of Fig. 3, where the core of the prominence is manifested as bright knots around the 225th pixel from the bottom of the slit. The temporal evolution of the line intensity along the slit for the warmer line, S III/Si III $1113 \AA$, is presented in the middle panel, and the corresponding Dopplergram evolution is plotted in the right panel. The siphon flow is also discerned in the intensity maps (clearly, in the middle panel, and slightly, in the left panel of Fig. 3), as well as the S III/Si III Dopplergram indicated by the nearly vertical streaks, moving from the upper part, to the lower part, of the slit. We observed three surges with the SUMER slit, as indicated in the middle panel. Comparison with the Dopplergram reveals that the surges initially displayed a red shift, which then turned to blue shift.

The predominant red shift of the siphon flow implies that the strong cool flow was moving away from the observer until 21:30 UT, with a line of sight velocity of about $24 \mathrm{~km} \mathrm{~s}^{-1}$. At 21:30 UT, the localised prominence, as well as the large-scale siphon flow (seen in the S III/Si III map), started to oscillate, as revealed by the alternation of the red and blue Doppler shifts. The oscillation of the prominence continued until 01:25 UT on 


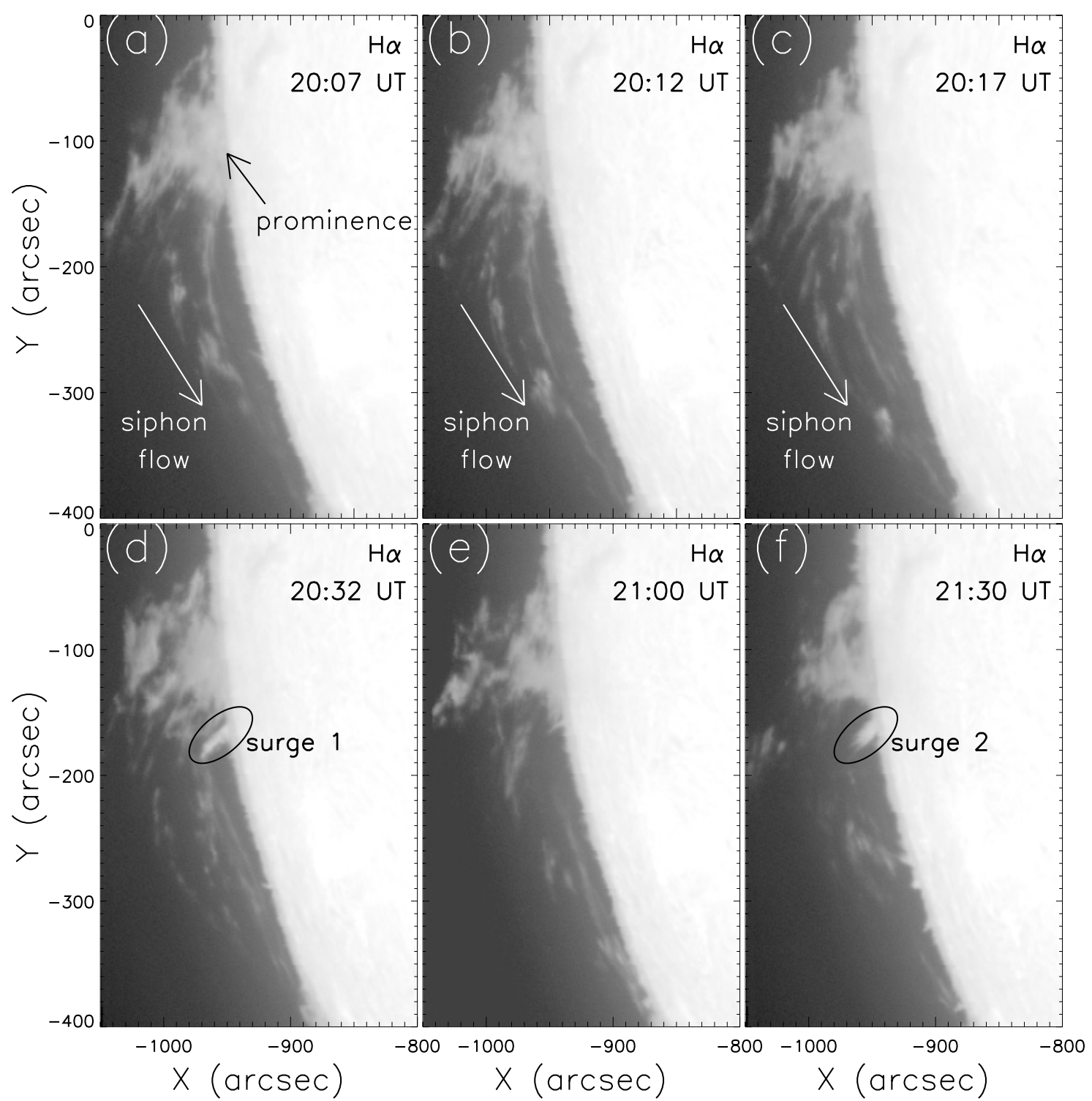

Fig. 2. A time sequence of $\mathrm{H} \alpha$ images, with the upper panels showing the siphon flow in a high cadence, and the lower panels showing the repetitive $\mathrm{H} \alpha$ surges.

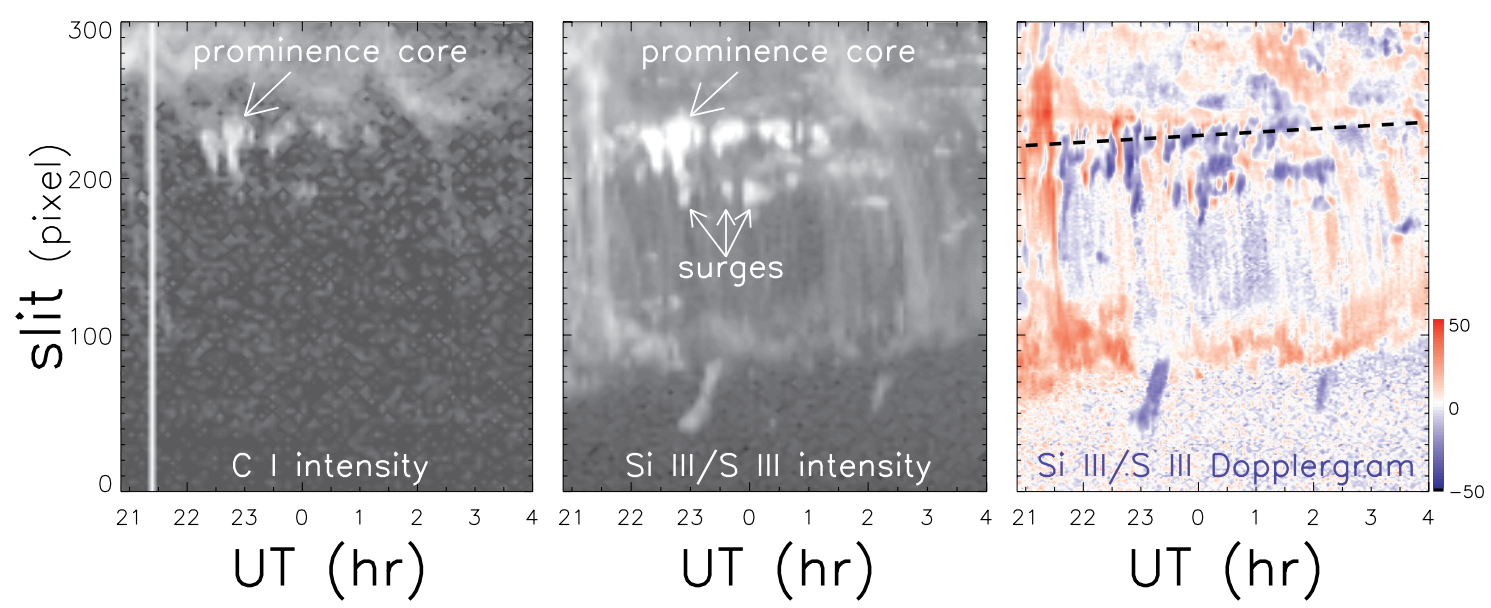

Fig. 3. Left panel: evolution of the C I $1118.45 \AA$ Antensity along the SUMER slit; Middle panel: same for S III/Si III $1113 \AA$ A Right panel: evolution of the Dopplergram along the SUMER slit observed at S III/Si III $1113 \AA$. The velocity evolution along the dashed cut-line in the right panel is analysed in Fig. 4. 

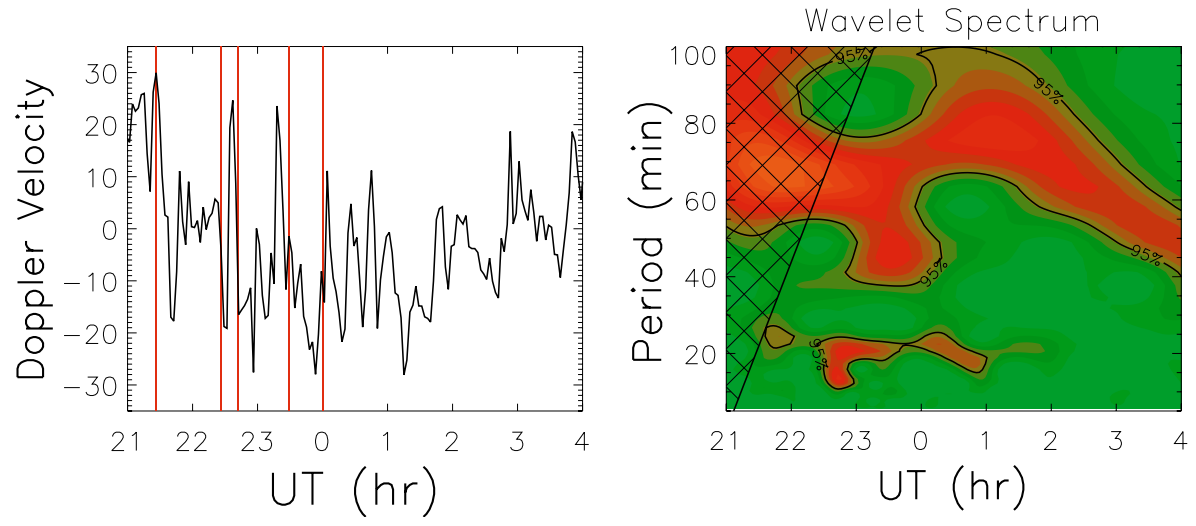

Fig. 4. Time variation of the Doppler velocity of the prominence core (left panel) and its wavelet spectrum (right panel). In the left panel, the red vertical lines mark the occurrence of the surges; The right panel indicates that the prominence oscillations present a period at $\sim 20 \mathrm{~min}$, as well as another one at $\sim 60 \mathrm{~min}$
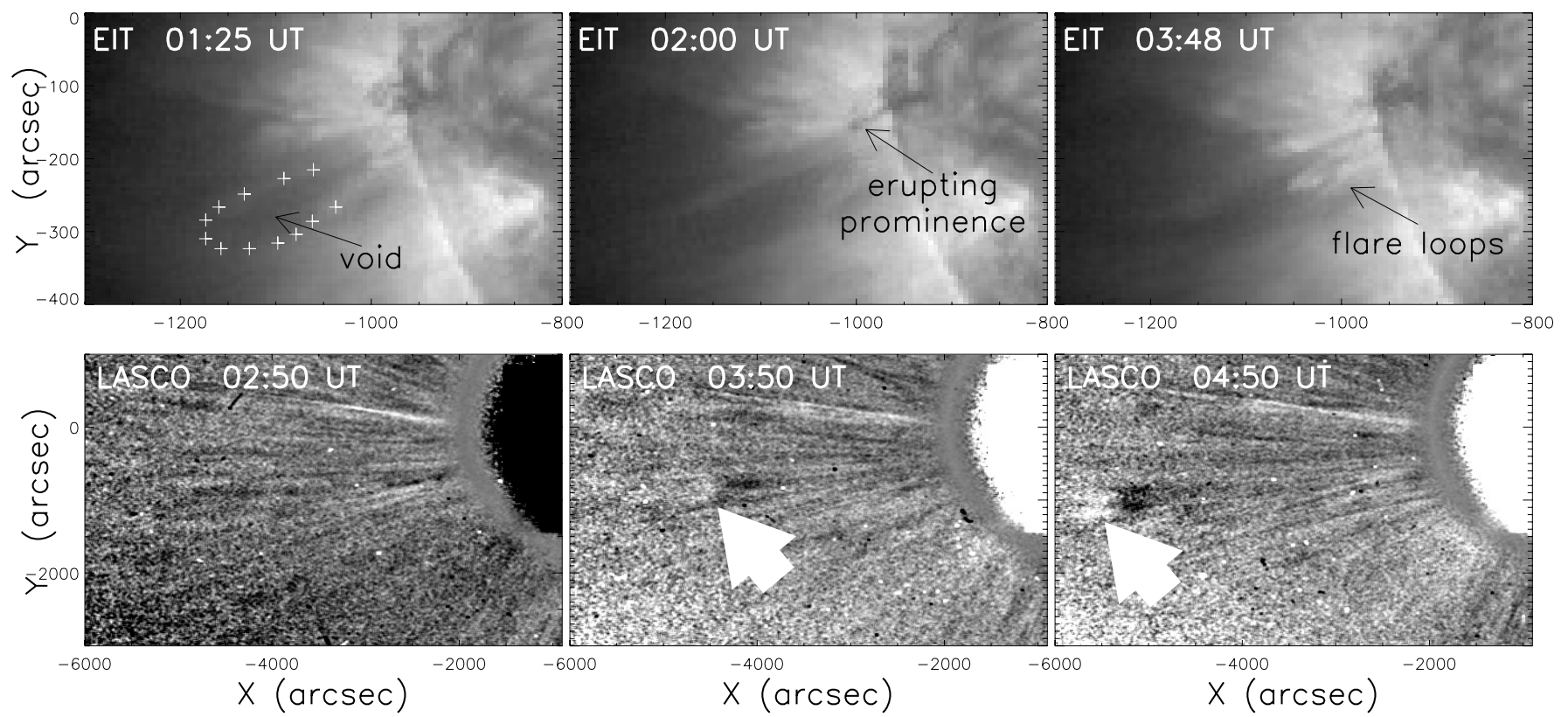

Fig. 5. Upper panels: SOHO/EIT $195 \AA$ Ailter images showing the expansion of the coronal loop (outlined by the plus signs), filament eruption, and the flaring; Lower panels: SOHO/LASCO images showing the prominence eruption as a blob-like CME.

Sep. 26. The oscillation period is estimated to be $\sim 20$ min by eye. In order to obtain the oscillation period more precisely, we performed a wavelet analysis of the Doppler velocity time series of the prominence core near the 225th pixel from the bottom of the slit. Figure 4 shows the Doppler velocity evolution in the left panel and its wavelet spectrum in the right panel, which is obtained by the method described in Torrence \& Compo (1998). Note that since the centroid of the prominence was shifting slowly to the north, the velocity evolution in the left panel of Fig. 4 is taken along the dashed line in the right panel of Fig. 3. The red regions indicate the locations of the highest power, and the cross-hatched region corresponds to the cone of influence. The wavelet spectrum confirms the $20 \mathrm{~min}$ period, and reveals another longer period, around $60 \pm 20 \mathrm{~min}$. After 01:25 UT, the prominence disappeared from the slit, which is suggested by the absence of bright knots in the left panel of Fig. 3. Note that Fig. 3 indicates that a weak S III/Si III siphon flow persisted all the time throughout the event, contrary to the $\mathrm{H} \alpha$ and $\mathrm{C}$ I siphon flows, which were not visible after 21:32 UT on Sep. 25. We also wish to mention that only a portion of the long prominence erupted, and the rest remained intact even after the eruption.

In order to investigate the later evolution of the prominence, a time sequence of the EUV $195 \AA$ images from SOHO/EIT and running difference white-light images from the LASCO C2 coronagraph are displayed in the upper and lower panels of Fig. 5, respectively. A careful examination of the EIT $195 \AA$ movie reveals that, starting at least from 22:00 UT on Sep. 25, an elongated coronal loop near the prominence was seen to be rising. At 01:25 on Sep. 26, the coronal loop, embedding a void, expanded to a height of about 250" above the solar limb (the coronal loop is outlined by the plus signs and the void is marked by the arrow in the upper left panel of Fig. 5). Subsequently, the prominence was lifted up, following the expansion of the coronal loop. At 02:00 UT (upper middle panel), the prominence significantly deviated from its original position. At 03:48 UT (upper right panel), the prominence was out of the field of view of SOHO/EIT, while flaring loops became visible below the expanding coronal loop mentioned above after 02:36 UT. The flare was so weak that it left no signature in the GOES 1-8 $\AA$ light curve.

At 02:50 UT on Sep. 26, we discerned a bright feature in the LASCO C2 field of view slightly south of the equatorial plane, as shown by the lower left panel of Fig. 5. Later, a clearly visible blob was seen to move almost radially, as indicated by the white arrows in the lower middle and right panels. Owing to the running difference method, a dark region immediately follows the bright blob. The bright blob looks like a typical CME core. 


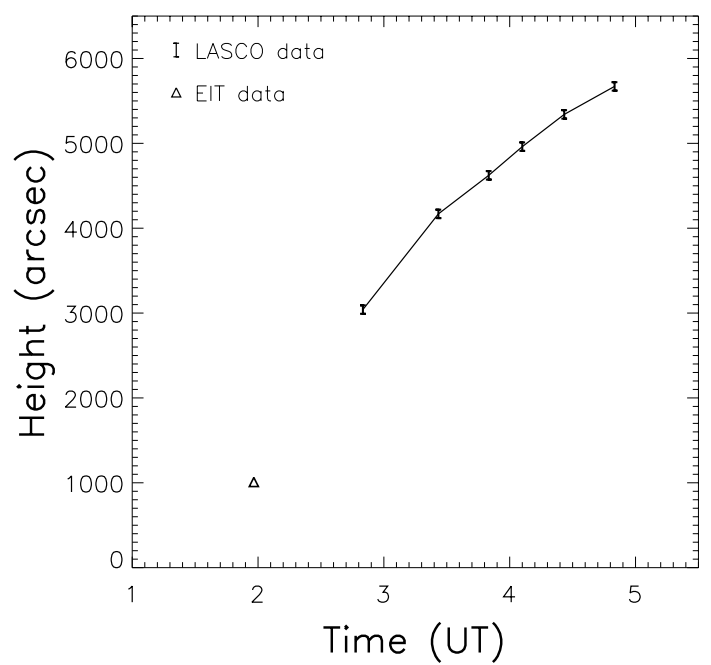

Fig. 6. Height-time plot of the EUV prominence (triangle point) and the white-light blob CME (error bars connected by solid lines).

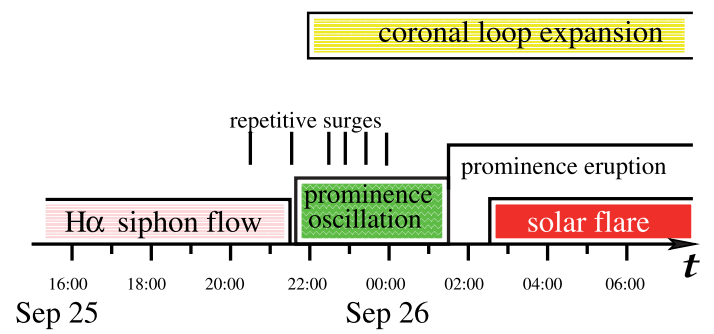

Fig. 7. Timeline of all phenomena associated with the prominence eruption.

In this small CME event, we saw no clear frontal loop, probably due to the limited instrumental sensitivity.

In Fig. 6, we plotted the heliocentric heights of the erupting prominence seen by SOHO/EIT (only one data point) and the erupting blob observed by SOHO/LASCO at various times. We found that the erupting velocity of the white-light blob was $\sim 216 \mathrm{~km} \mathrm{~s}^{-1}$, and the EUV prominence was roughly aligned with the height-time profile of the white-light blob, confirming that the erupting blob was most likely the erupting prominence.

\section{Discussions}

\subsection{Global picture}

Based on the analysis of all available observational data, the whole process is summarised in Fig. 7, and described as follows: Starting earlier than 16:00 UT on Sep. 25, a strong $\mathrm{H} \alpha$ siphon flow from the proximity of the prominence to a site $270^{\prime \prime}$ to the south was sustained until 21:32 UT. During this period, there was one surge eruption near the prominence at $\sim 20: 32$ UT. A second surge erupted at the same site at $\sim 21: 26 \mathrm{UT}$, and almost simultaneously, the prominence began to oscillate, with a period of $\sim 20 \mathrm{~min}$. At the same time, an elongated coronal loop above the prominence began to rise. The prominence oscillation continued for $4 \mathrm{~h}$, during which time repetitive surges erupted at the same site. Starting at $\sim 01: 25$ UT on Sep. 26 , the prominence was pulled up and ejected as a blob-like CME. Below the erupting prominence, flaring loops appeared near the solar surface after 02:36 UT.

The late evolution of the event, from the coronal loop expansion to the eruption of the prominence, as well as the ensuing solar flare, fits well into the standard model for CME/flare
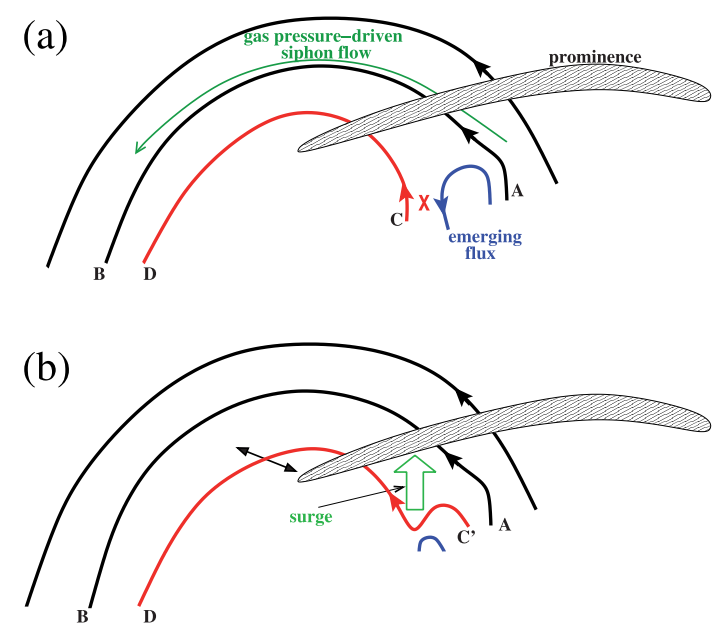

Fig. 8. Sketch of the whole process, where the thick lines represent magnetic field. Panel a): emerging flux appears outside the filament channel, which drives the siphon flow near point A due to increased gas pressure and $\mathrm{H} \alpha$ surges near point $\mathrm{C}$ due to magnetic reconnection. Panel $\mathbf{b}$ ): the kinked magnetic loop $C^{\prime} \mathrm{D}$ after reconnection triggers the oscillations of the magnetic loops, along with the prominence, and the expansion of the coronal loops, which finally pulls the prominence to erupt.

events, i.e, owing to some kind of instability or loss of equilibrium, a coronal magnetic loop rises, and an embedded flux rope, if one is present, subsequently also rises, as the forces holding it down are reduced. As a result, a current sheet forms below the flux rope. Magnetic reconnection in the current sheet leads to the formation of flaring loops below the reconnection point and the eruption of the flux rope above the reconnection point (see Shibata 2005, for a historic review). However, in this event it remains unclear how the initial loss of equilibrium was triggered, an essential problem in CME research, since this event occurred near the limb, where the quality of the local magnetogram was extremely poor, and multiwavelength observations of the low corona suffer seriously from the foreshortening effect. Besides, a part of the activity was occulted by the solar limb. Nevertheless, some clues can be gleaned on the basis of previous research. It has been established that a siphon flow is driven by enhanced gas pressure at one end of a coronal loop (Meyer \& Schmidt 1968), whereas $\mathrm{H} \alpha$ surges are generated by magnetic reconnection between emerging flux and the large-scale coronal field (Kurokawa \& Kawai 1993; Yokoyama \& Shibata 1995). Therefore, we can construct the following self-consistent paradigm for this event, which is illustrated in Fig. 8: coronal magnetic loops $\mathrm{AB}$ and $\mathrm{CD}$ are field lines straddling over the prominence and helping to keep it stable. Magnetic flux emerges just outside the filament channel with the polarity orientation drawn in the upper panel. Its inner (left) leg has opposite magnetic polarity from the background and can therefore trigger reconnection. The other (right) leg has the same polarity as the background. Therefore, the merging flux squeezes the plasma against the magnetic loop $\mathrm{AB}$, which enhances the plasma density as well as the gas pressure near point $\mathrm{A}$. The increased gas pressure would drive a siphon flow along the magnetic field line from point A to point B. Such a process may commence as soon as the new flux emerges above the surface. Note that, since the long prominence was straddling over the solar east limb, and the fresh magnetic flux may have emerged behind the limb (this is supported by the fact that the $\mathrm{H} \alpha$ surges did not show any signature on the disc), the siphon flow was apparently seen to be running slightly below the prominence on the projected plane. 
Between the emerging flux and the field line CD, a current sheet forms since the left leg of the emerging flux has the magnetic polarity opposite to the background (Solanki et al. 2003). After some time, e.g., when the current density (or the electron drift speed in the electric current) exceeds a threshold, reconnection is triggered at point " $\mathrm{X}$ ", which would drive an $\mathrm{H} \alpha$ surge. After the reconnection, the field line $\mathrm{CD}$ becomes $\mathrm{C}^{\prime} \mathrm{D}$. As demonstrated by Chen \& Shibata (2000), the reconnected field line $C^{\prime} D$ will expand in response to the magnetic restructuring. However, in their 2D numerical simulations, the emerging flux and the preexisting coronal loops are in the same plane, so there is no kink perturbation perpendicular to the loop plane. If the emerging flux is inclined to the CD loop plane, as plotted in Fig. 8, the newly-reconnected loops obtain a strong kink perturbation. In this way, the magnetic loop $\mathrm{C}^{\prime} \mathrm{D}$ would oscillate in the direction perpendicular to the loop plane, as indicated by the doubleheaded arrow in the lower panel of Fig. 8. The oscillation of the field line would drag the prominence to oscillate in the same way. As demonstrated by Chen \& Shibata (2000), the expanding coronal loop $\mathrm{C}^{\prime} \mathrm{D}$ finally does not hold it down so strongly any more, so the prominence rises. The following formation and disruption of a current sheet are well described by the standard model, which was explained at the beginning of this paragraph.

Therefore, we make a conjecture that the emerging flux drives the siphon flow and the reconnection with the pre-existing coronal magnetic field, which leads to the formation of the $\mathrm{H} \alpha$ surges, triggering the oscillation and later eruption of the prominence. The fact that reconnection-favoured emerging flux can trigger the filament/prominence eruption has been verified by Feynman \& Martin (1995; see also Innes et al. 1999) and explained by Chen \& Shibata (2000). The observations presented here emphasise that the magnetic reconnection, which occurs between the emerging flux and the pre-existing coronal field in the CME triggering process, takes place intermittently, rather than continually, as implied by the repetitive behaviour of the $\mathrm{H} \alpha$ surges. Such a repetitive behaviour of $\mathrm{H} \alpha$ surges, with a period of $\sim 1 \mathrm{~h}$, was reported by Schmieder et al. (1984). The repetitive reconnection might be modulated by MHD waves in a way similar to that proposed by Chen \& Priest (2006). The repetitive reconnection can also account for the occurrence of the typeIII radio-burst group several hours before the CME as found by Jackson et al. (1978).

\subsection{Prominence oscillations as a precursor of CMEs}

Prominence oscillations were first observed by Ramsey \& Smith (1966). Since then, considerable progress has been made due to both imaging and spectroscopic observations. Prominence oscillations can be divided into large-amplitude type with velocity amplitudes of the order of $20 \mathrm{~km} \mathrm{~s}^{-1}$ (e.g., Ramsey \& Smith 1966) and small-amplitude type with velocity amplitudes of the order of 2-3 $\mathrm{km} \mathrm{s}^{-1}$ (e.g., Thompson \& Schmieder 1991). Concerning the period $(P)$, they can also be categorised into three types (Molowny-Horas et al. 1997): short type $(P<$ 5 min, e.g., Thompson \& Schmieder 1991), intermediate type ( $P \sim$ 6-20 min, e.g., Bocchialini et al. 2001), and long type $(P \sim 40$ min-2 h, e.g., Terradas et al. 2002). After identifying the oscillation mode, the properties of the oscillations, including the period, damping time, wavelength, and so on, can then be used to diagnose both thermal and magnetic parameters of the prominence (see Roberts 2000; Oliver \& Ballester 2002, for reviews).

Prominence oscillations can be caused by either an internal or external agent (Vršnak 1993). The internal agent might be an instability of the prominence. For example, Malville \& Schindler (1981) interpreted a prominence oscillation in terms of kink instability. The external agent might be Moreton waves from another active region (e.g., Ramsey \& Smith 1966), photospheric 5-min and chromospheric 3-min oscillations (e.g., Blanco et al. 1999), or photospheric convection (e.g., Malville 1968). The initiation of a CME is also a kind of perturbation, which should trigger the oscillation of the prominence/filament in the CME source region (note that almost $90 \%$ of CMEs are associated with prominences, St Cyr \& Webb 1991). For example, Malville \& Schindler (1981) were the first to find a prominence that oscillated for at least $90 \mathrm{~min}$ prior to the onset of a limb flare. From the theoretical point of view, it is also reasonable to see prominence oscillations before eruption. In the initiation models regarding the photospheric shearing and convergent motions (e.g., Forbes \& Priest 1995), a kink instability would be responsible for the triggering of the prominence oscillations; in the tether-cutting initiation model (Moore \& Labonte 1980), the first-step reconnection of the internal magnetic field would induce a strong perturbation to the prominence. Accordingly, in the emerging flux trigger mechanism (Chen \& Shibata 2000), magnetic reconnection between the emerging flux and the preexisting magnetic field would impose a strong kink perturbation to the magnetic field around the prominence. The lateral kink, as depicted in Fig. 8 and analysed here, would drive horizontal string-type oscillations of the coronal magnetic field and the filament. In the 2D numerical simulations of Chen \& Shibata (2000), there was no $B_{z}$ in the magnetic configuration. We have performed another simulation, with $B_{z}$ being introduced to the emerging flux. The preliminary results indicate that the flux rope, which is believed to hold a filament at the bottom, does show string-type oscillations in the direction of the magneticneutral line. Therefore, we expect to see prominence oscillations in the initiation phase of a CME no matter what the initiation mechanism is. That is to say, prominence oscillations can be considered as another precursor of CMEs.

Since the moving direction of the string-mode oscillations is in the horizontal plane and perpendicular to the filament thread, they are best observed by spectroscopic instruments like SUMER when the filament is above or near the solar limb and the filament thread is inclined to the line of sight. Near the solar disc centre, such a string-mode oscillation manifests itself as a spatial displacement. Consider that the period is $\sim 20 \mathrm{~min}$ and that the velocity amplitude is $\sim 10 \mathrm{~km} \mathrm{~s}^{-1}$, the amplitude of the displacement is $\sim 1910 \mathrm{~km}$, or $\sim 2.6$ arcsec, which could be resolved with high-resolution imaging observations.

Prominences often oscillate, even in the quiescent state (e.g., Régnier et al. 2001), since perturbations such as MHD waves from sporadic eruptions, persistent $p$-mode oscillations and convective motions in the photosphere, are ubiquitous in the solar atmosphere. No prominence eruption follows these kinds of oscillations. However, for these non-eruptive oscillations, the damping time is usually between 1 and 3 times the corresponding period (e.g., Tsubaki \& Takeuchi 1986; Wiehr et al. 1989; Molowny-Horas et al. 1997), whereas, our analysis of the 2000 September 26 event indicates that the prominence oscillations lasted $\sim 4 \mathrm{~h}$, almost 12 times the corresponding period, before the final eruption. The reason is that the excitation during the CME trigger phase is continuous, rather than just a short pulse. The occurrence of the repetitive $\mathrm{H} \alpha$ surges in our event, at intervals of about $60 \mathrm{~min}$, strongly suggests that the reconnection between the emerging flux and the pre-existing magnetic field proceeds in an intermittent, probably quasi-periodic, way (see the timeline in Fig. 7). The repetitive reconnection keeps shaking 
the magnetic loops around the prominence, leading to the unusually long-time oscillations of the prominence. This is supported by the almost one-to-one correspondence of the $\mathrm{H} \alpha$ surges and the occurrences of the increased oscillation amplitude as shown in the left panel of Fig. 4. Between the occurrence of the surges, the oscillation amplitude did show fast damping. In this sense, different from the 20 -min period of the prominence oscillations, which is the intrinsic period of the prominence, the 60-min period in the wavelet spectrum of the prominence oscillation reflects the period of the repetitive reconnection, which serves as an external driving agent for the oscillations. Actually, the prominence oscillation can continue even in the eruption phase, which was discovered by Isobe \& Tripathi (2006). They noticed in their event that the prominence oscillation was also caused by emerging flux. The results of this work invite investigation of more prominences to learn which fraction of them oscillate prior to eruption.

Acknowledgements. The authors thank MLSO and Haimin Wang at BBSO for providing $\mathrm{H} \alpha$ data. Thanks are also due to $\mathrm{H}$. Kurokawa for discussions about surges and to the referee for the comments. The research is supported by the Chinese foundations NCET-04-0445, FANEDD (200226), 2006CB806302, and NSFC (10221001, 10333040 and 10403003). SOHO is a project of international cooperation between ESA and NASA.

\section{References}

Blanco, S., Bocchialini, K., Costa, A., et al. 1999, Sol. Phys., 186, 281 Bocchialini, K., Costa, A., Domenech, G., et al. 2001, Sol. Phys., 199, 133 Brueckner, G. E., Howard, R. A., Koomen, M. J., et al. 1995, Sol. Phys., 162, 357

Chen, P. F., \& Priest, E. R. 2006, Sol. Phys., 238, 313

Chen, P. F., \& Shibata, K. 2000, ApJ, 545, 524

Chertok, I. M., Kahler, S., Aurass, H., \& Gnezdilov, A. A. 2001, Sol. Phys., 202, 337

Delaboudinière, J.-P., Artzner, G. E., Brunaud, J., et al. 1995, Sol. Phys., 162, 291

Feynman, J., \& Martin, S. F. 1995, J. Geophys. Res., 100, 3355
Forbes, T. G., \& Priest, E. R. 1995, ApJ, 446, 377

Gopalswamy, N., Mikić, Z., Maia, D., et al. 2006, Space Sci. Rev., 123, 303

Harrison, R. A., Waggett, P. W., Bentley, R. D., et al. 1985, Sol. Phys., 97, 387

Innes, D. E., Inhester, B., Srivastava, N., et al. 1999, Sol. Phys., 186, 337

Isobe, H., \& Tripathi, D. 2006, A\&A, 449, L17

Jackson, B. V., Sheridan, K. V., Dulk, G. A., \& McLean, D. J. 1978, Proc. Astron. Soc. Austr., 3, 241

Kurokawa, H., \& Kawai, G. 1993, IAU Colloq. 141: The Magnetic and Velocity Fields of Solar Active Regions, 46, 507

Lantos, P., Kerdraon, A., Rapley, G. G., \& Bentley, R. D. 1981, A\&A, 101, 33

Malville, J. M. 1968, Sol. Phys., 4, 323

Malville, J. M., \& Schindler, M. 1981, Sol. Phys., 70, 115

Martin, S. F. 1980, Sol. Phys., 68, 217

Meyer, F., \& Schmidt, H. U. 1968, Mitteilungen der Astronomischen Gesellschaft Hamburg, 25, 194

Molowny-Horas, R., Oliver, R., Ballester, J. L., \& Baudin, F. 1997, Sol. Phys., 172,181

Moore, R. L., \& Labonte, B. J. 1980, Solar and Interplanetary Dynamics, 91, 207

Oliver, R., \& Ballester, J. L. 2002, Sol. Phys., 206, 45

Ramesh, R., \& Sundaram, G. A. S. 2001, Sol. Phys., 202, 355

Ramsey, H. E., \& Smith, S. F. 1966, AJ, 71, 197

Régnier, S., Solomon, J., \& Vial, J. C. 2001, A\&A, 376, 292

Roberts, B. 2000, Sol. Phys., 193, 139

Schmieder, B., Mein, P., Martres, M. J., \& Tandberg-Hanssen, E. 1984, Sol. Phys., 94, 133

Shibata, K. 2005, IAU Symp., 226, 241

Solanki, S. K., Lagg, A., Woch, J., Krupp, N., \& Collados, M. 2003, Nature, 425, 692

St. Cyr, O. C., \& Webb, D. F. 1991, Sol. Phys., 136, 379

Sterling, A. C., \& Moore, R. L. 2005, ApJ, 630, 1148

Sterling, A. C., Harra, L. K., \& Moore, R. L. 2007, ApJ, 669, 1359

Terradas, J., Molowny-Horas, R., Wiehr, E., et al. 2002, A\&A, 393, 637

Thompson, W. T., \& Schmieder, B. 1991, A\&A, 243, 501

Torrence, C., \& Compo, G. P. 1998, Bull. Am. Meteorological Soc., 79, 61

Tsubaki, T., \& Takeuchi, A. 1986, Sol. Phys., 104, 313

Vrsnak, B. 1993, Hvar Observatory Bulletin, 17, 23

Wen, Y.-Y., Wang, J.-X., \& Zhang, Y.-Z. 2007, Chinese J. Astron. Astrophys., 7, 265

Wiehr, E., Balthasar, H., \& Stellmacher, G. 1989, Hvar Obs. Bull., 13, 131

Wilhelm, K., Curdt, W., Marsch, E., et al. 1995, Sol. Phys., 162, 189

Yokoyama, T., \& Shibata, K. 1995, Nature, 375, 42 